\title{
ANÁlise de Correlações entre as SÉries do Módulo de Velocidade e a Direção do Vento Para Diferentes localizaÇões Pelo Nordeste Brasileiro por APliCaÇão da TéCNICA de COERÊNCIA WAVELET
}

\author{
VAlciano C. Gurgel ${ }^{1}$, Francisco E. A. Leite ${ }^{2}$, Marcos V. C. Henriques ${ }^{2}$. \\ 1. Bolsista de Iniciação Científica PIBIC - CNPq \\ Grupo de Análise e Processamento de Sinais e Imagens - GAPSI \\ Universidade Federal Rural do Semiárido, UFERSA \\ Campus Angicos, 59515-000, RN, Brasil \\ E-mail: Valciano camilolyahoo.com.br \\ 2. Departamento de Ciências Exatas, Tecnológicas e Humanas- DCETH \\ Universidade Federal Rural do Semiárido, UFERSA \\ Campus Angicos, 59515-000, BRASIL- RN. \\ E-mails: edcarloseufersa.edu.br; vini.rnegmail.com
}

\begin{abstract}
Resumo - O presente trabalho tem por objetivo apresentar a constatação de um padrão de semelhanças encontradas nas correlações entre as séries temporais meteorológicas do módulo da velocidade e da direção do vento em vários pontos geográficos espalhados por alguns estados nordestinos brasileiros. Os dados trabalhados foram recolhidos por plataformas de coleta de dados (PCDs) mantidas pelo Instituto Nacional de Meteorologia (INMET), espalhadas no interior e litoral do Brasil. O estudo é embasado na teoria matemática das Wavelets. Especificamente, a técnica utilizada aqui é conhecida por Coerência Wavelet. Essa técnica realiza uma transformação sobre os dados reais para o espaço das wavelets utilizando dois parâmetros: tempo e escala. Os resultados podem ser visualizados por meio de escalogramas de cores nos quais se denotam a ocorrência de coincidência no tempo e escala como também a fase entre os sinais. Esta pesquisa destaca duas constatações: a de que existe forte correlação entre o módulo da velocidade e a direção do vento no período analisado; e identifica um padrão de semelhança entre as correlações que pode ser relacionada à localização das estações de coleta. A semelhança entre estações é evidente na comparação visual entre o tipo de correlação encontrado e a região da localização geográfica da plataforma de coleta. Em analogia simplificada destacam-se as correlações e semelhanças encontradas nas regiões litorâneas daquelas encontradas nas regiões interioranas. A análise pode ser estendida levando em conta outras variáveis que certamente influenciam na ocorrência das que foram estudadas neste artigo.
\end{abstract}

Palavras-chave — séries temporais, transformada wavelet, dados meteorológicos, correlação.

\section{Introdução}

Em muitas áreas do conhecimento os sinais e series temporais são encontrados [4]. A ferramenta mais difundida entre os profissionais para processamento de sinais é a Transformada de Fourier [3]. Se o sinal apresenta variações no tempo de suas propriedades estatísticas, então ele é dito não estacionário. Tentou-se analisar processos não estacionários utilizando a Transformada "Janelada" de Fourier (Windowed Fourier Transform ou Short-Time Fourier Transform), esta proposta pioneira foi desenvolvida por Gabor [6]. Porém, a eficiência desse método é limitada pelo tamanho da janela invariável, tornando difícil a análise de altas e baixas frequências simultaneamente [2].

Portanto, para processos não estacionários as metodologias existentes ainda são razoavelmente precárias [4]. A Transformada Wavelet apresenta melhorias para esta deficiência uma vez que ela utiliza parâmetros variáveis que são ajustáveis a cada trecho da função analisada.

\section{Transformada Wavelet}

A Transformada Wavelet mostra-se como uma nova ferramenta que diferentemente da Transformada de Fourier permite o tratamento de dados não estacionários [5]. Ela é pensada como uma ferramenta contemporânea com usos relevantes em diversas áreas. Potencialmente, o mais atrativo para aplicações das Wavelets é o processamento de sinais [3], sendo amplamente utilizada também em estatística (estimação do espectro de processos estacionários; estimação do espectro dependente do tempo de processos não estacionários), meteorologia (análise de fluxos turbulentos), codificação e compressão de imagens, música, processos estocásticos, certas áreas da matemática, economia, oceanografia, geofísica, etc. [4]. Em áreas que tradicionalmente utilizam análise de Fourier, o uso das funções Wavelets podem significar processamento mais rápido e valiosas informações adicionais [6].

A característica mais importante das Wavelets é sua capacidade e obter localização tanto no tempo (ou espaço) quanto na frequência [5]. Nesta 
transformada faz-se uso do sistema de funções Wavelets como funções de análise para obter-se uma representação no espaço das Wavelets [2]. No processo de transformação as funções básicas são definidas por dois parâmetros: posição e escala. É interessante que as funções funcionam como uma janela que diminui para capturar componentes de alta frequência e aumenta para capturar as de baixa frequência [4]. Então dizemos que as Wavelets conseguem captar o comportamento local ou global de um sinal [5].

As funções Wavelets apresentam as seguintes propriedades [4]:

$$
\int_{-\infty}^{+\infty} \psi(t) d t=0
$$

I. A condição de admissibilidade (1) impõe que as Wavelets tenham média nula e caráter oscilatório.

$$
\int_{-\infty}^{+\infty}|\psi(t)|^{2} d t \triangleleft 0
$$

II. Indica que as wavelets possuem energia finita.

Sendo assim, a seguinte equação define uma família de funções Wavelets [2]:

$$
\psi_{a, b}(t)=\frac{1}{\sqrt{|a|}} \psi^{*} \frac{(t-b)}{a}
$$

Onde $a$ e $b$ são números reais que representam os parâmetros tempo e escala, respectivamente, sendo $a>0$. O subscrito (*) representa o conjugado complexo. $\Psi$ é chamada de wavelet-mãe [7].

\section{Transformada Wavelet Contínua}

A Transformada Wavelet Contínua de uma função $f(t)$ é calculada por meio do produto interno da própria função com a wavelet-mãe, na escala $a$ e posição $b$. Para uma determinada série denotada por $x$ temos que a sua transformada wavelet continua é dada por:

$$
C x_{(a, b)}=\int_{-\infty}^{+\infty} f(t) \psi_{a, b}^{*}(t) d t
$$

\section{Coerência Wavelet}

Antes de haver a técnica da Coerência Wavelet já existia o Espectro Cruzado Wavelet que deu origem à definição matemática da coerência. Ver detalhes em [3]. O espectro é calculado entre dois sinais, $x$ e $y$, através da seguinte equação:

$$
C x y_{(a, b)}=S\left(C x_{(a, b)}^{*} C y_{(a, b)}\right)
$$

O subscrito (*) representa o conjugado complexo da transformada, e $S$ é um operador de suavização da operação no tempo e na escala. Mais detalhes sobre o operador em [7].

Agora podemos definir a Coerência Wavelet entre duas series $x$ e $y$ através da seguinte formula:

$$
\operatorname{Cohxy}_{(a, b)}=\frac{S\left(C x_{(a, b)}^{*} C y_{(a, b)}\right)}{\sqrt{S\left(\left|C x_{(a, b)}\right|^{2}\right)} \sqrt{S\left(\left|C y_{(a, b)}\right|^{2}\right.}}
$$

A expressão (6) comporta séries temporais de valores reais e complexos.

\section{Resultados}

Analisou-se a correlação detectada entre as séries temporais meteorológicas: módulo de velocidade do vento, em metros por segundo $(\mathrm{m} / \mathrm{s})$, com a direção do vento, em graus (de zero a trezentos e sessenta no sentido horário de acordo com a convenção da rosa dos ventos).

Em cada estação foi aplicada a técnica Coerência Wavelet no par de séries, velocidade e direção do vento como foi citado. O resultado é apresentado de maneira gráfica em escalogramas de cores. O espaço geográfico abrangido pelas estações caracteriza dimensões significativas do espaço territorial brasileiro.

Os dados estudados foram adquiridos do banco de dados de domínio público disponibilizados no seguinte endereço eletrônico: http://www.inmet.gov.br/portal/index.php?r=bd mep/bdmep, do Instituto Nacional de Meteorologia (INMET) no qual se encontram disponíveis séries meteorológicas históricas com medidas diárias a partir de 1961 até o ano presente para 291 localidades. 


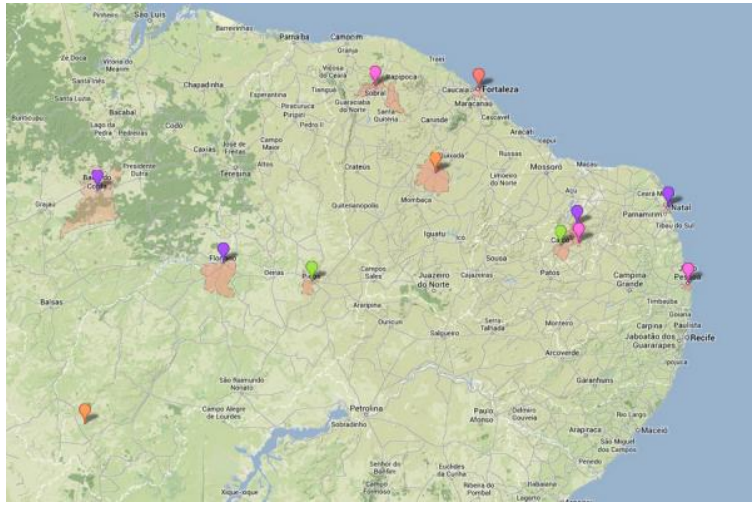

Figura 1: Localização geográfica das plataformas de coleta de dados no litoral e interior nordestino.

A tabela a seguir lista os nomes das plataformas que registraram os dados em estudo neste trabalho de acordo com o nome das cidades onde se encontram.

Tabela 1. Cidades onde se localizam as PCDs.

\begin{tabular}{|l|l|}
\hline 1. Barra do Corda & 7. Fortaleza \\
\hline 2. Caicó & 8. João pessoa \\
\hline 3. Caracol & 9. Natal \\
\hline 4. Cruzeta & 10. Picos \\
\hline 5. Florania & 11. Quixeramobim \\
\hline 6. Floriano & 12. Sobral \\
\hline
\end{tabular}

Os gráficos cartesianos das séries temporais são apresentados juntamente na mesma janela e demonstram o caráter não linear e as mudanças abruptas no comportamento dos sinais. O gráfico em azul é relativo ao módulo de velocidade do vento enquanto o gráfico em vermelho diz respeito à sua direção no mesmo instante de tempo. Trata-se de grandezas diferentes e, portanto, vale resaltar que possuem unidades diferentes. A intenção dê serem mostrados juntos é a visualização da diferença de fase entre os sinais.

As setas nos escalogramas representam a diferença de fase entre os sinais. Quando as setas apontam para o lado direito indica que naquele instante de tempo os sinais estão em fase. Já quando as setas apontam para o lado esquerdo indica que há diferença de fase com $180^{\circ}$. Para mais informações consultar [7].

A seguir são apresentados os escalogramas que representam as correlações wavelets entre as séries temporais em questão no espaço das wavelets com parâmetro de escala 1024 e 3000 observações ao longo de dois anos (tempo). A função wavelet-mãe utilizada é a Daubechies 3 por apresentar formato semelhantes aos dos sinais.
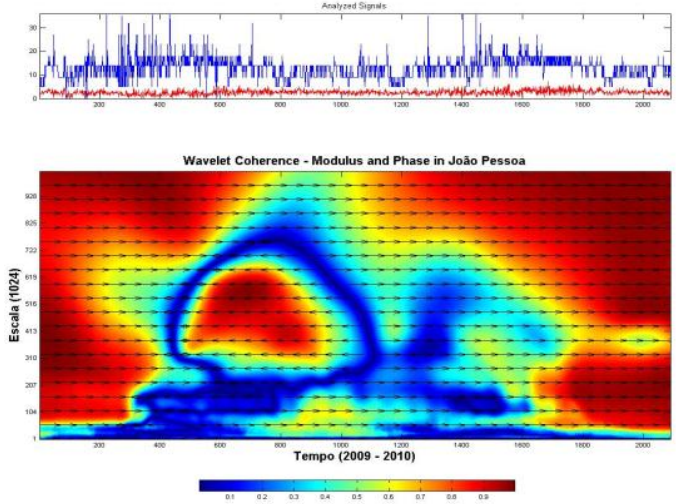

Figura 2: Correlação entre velocidade e direção do vento na PCD de João Pessoa
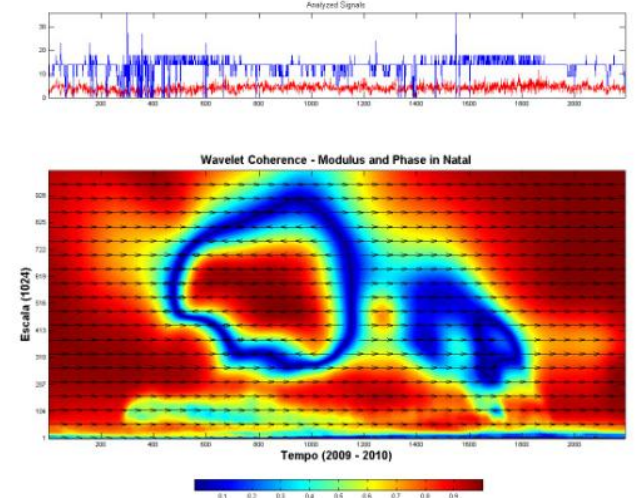

Figura 3: Correlação entre velocidade e direção do vento na PCD de Natal
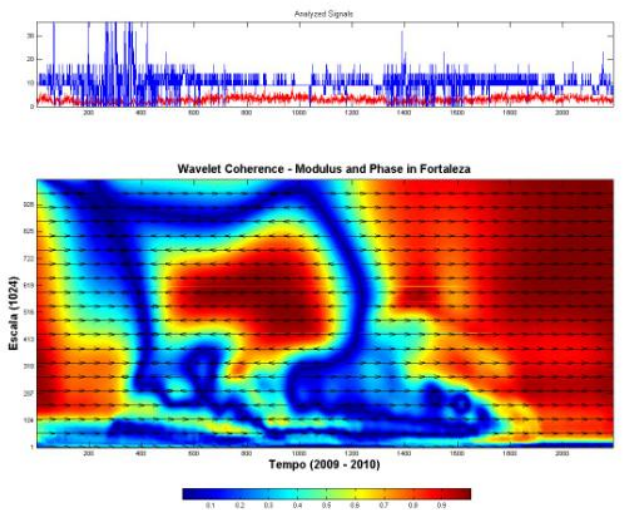

Figura 4: Correlação entre velocidade e direção do vento na PCD de Fortaleza 

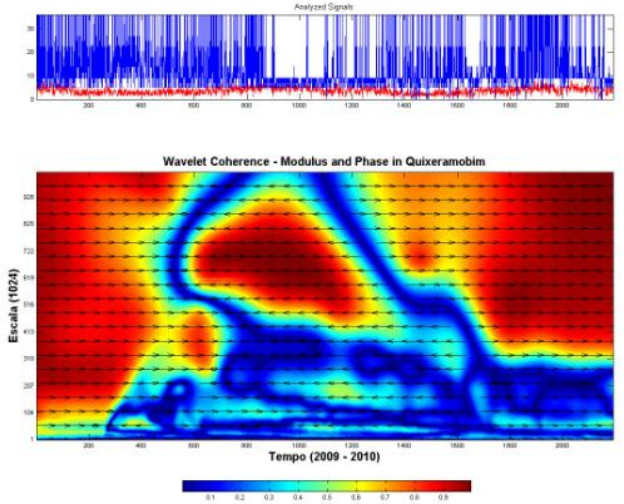

Figura 5: Correlação entre velocidade e direção do vento na PCD de Quixeramobim
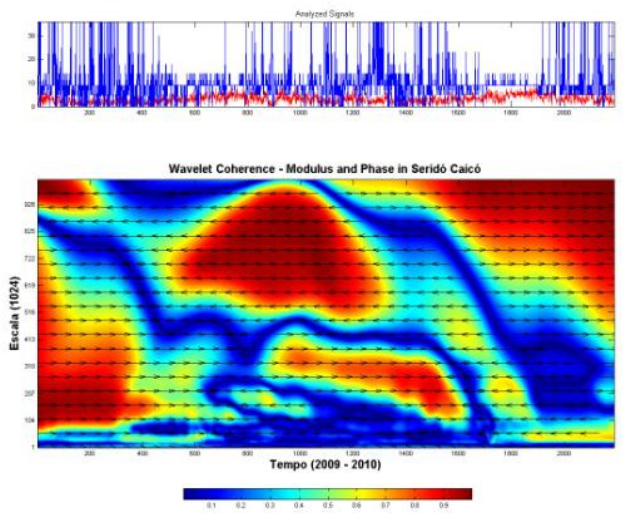

Figura 6: Correlação entre velocidade e direção do vento na PCD de Seridó Caicó
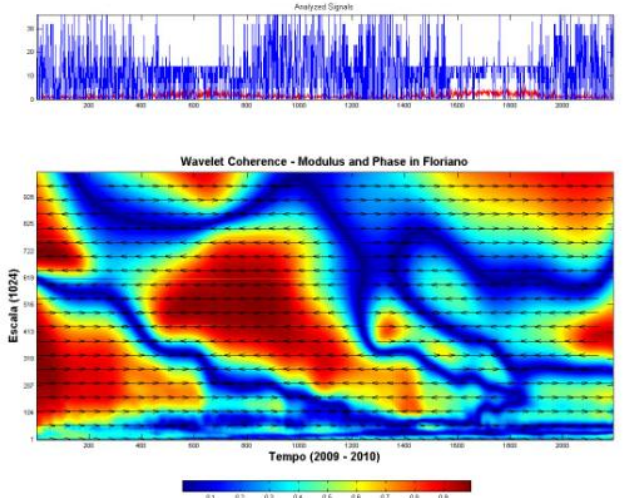

Figura 7: Correlação entre velocidade e direção do vento na PCD de Floriano
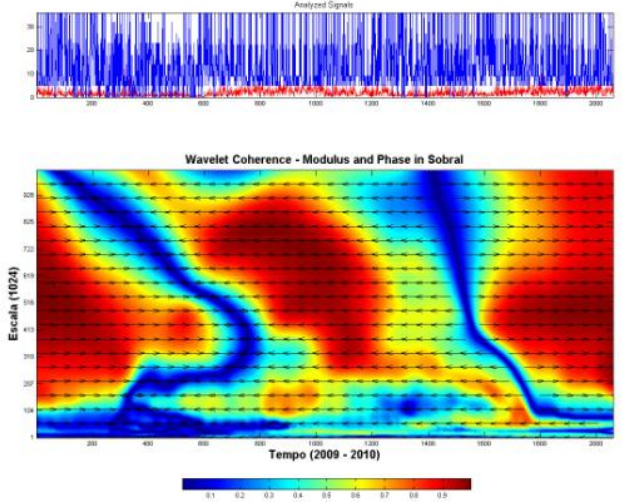

Figura 8: Correlação entre velocidade e direção do vento na PCD de Sobral
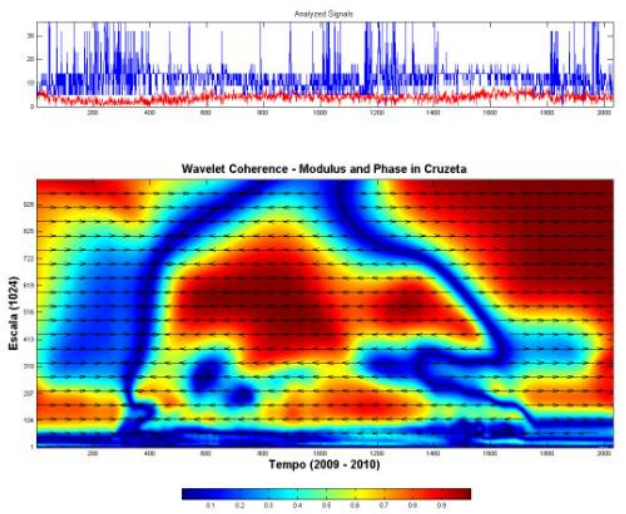

Figura 9: Correlação entre velocidade e direção do vento na PCD de Cruzeta
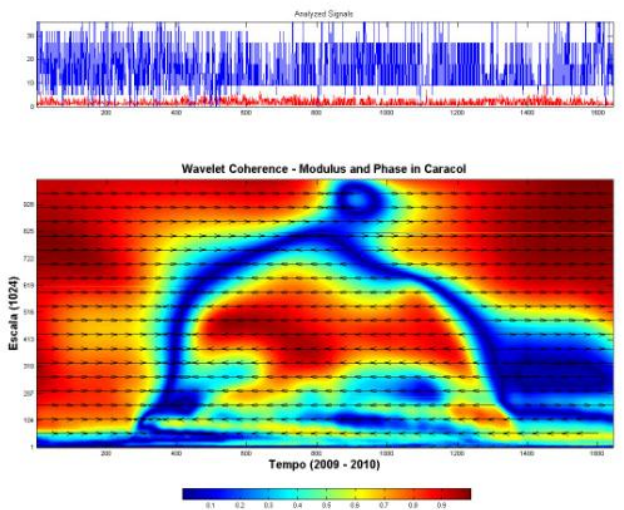

Figura 10: Correlação entre velocidade e direção do vento na PCD de Caracol 

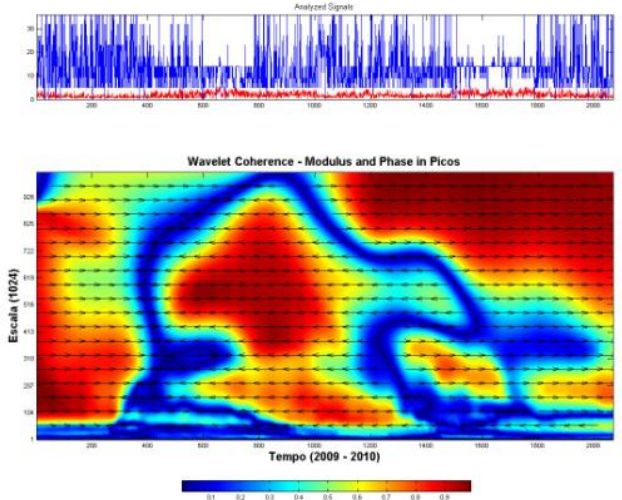

Figura 11: Correlação entre velocidade e direção do vento na PCD de Picos
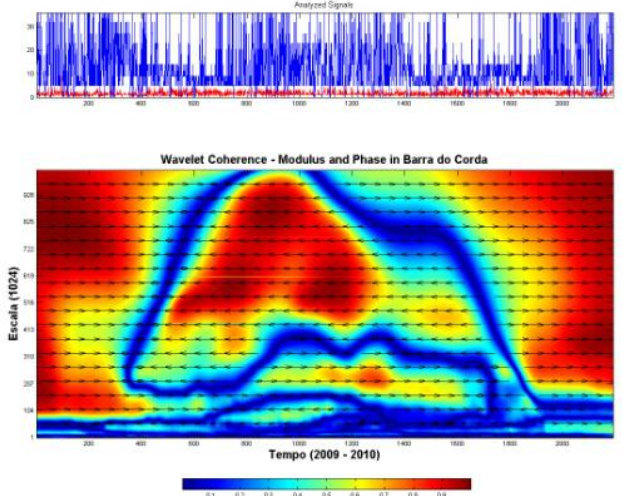

Figura 12: Correlação entre velocidade e direção do vento na PCD de Barra do Corda
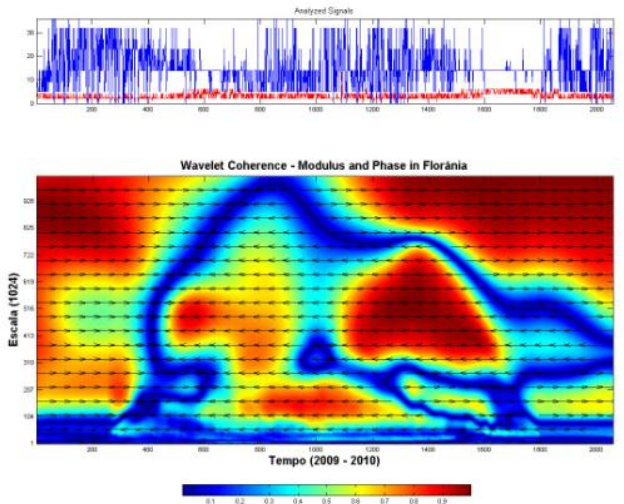

Figura 13: Correlação entre velocidade e direção do vento na PCD de Florania

\section{Conclusões}

Foi constatado que existe um padrão de correlação entre as grandezas trabalhadas no tempo e escala analisados. A interpretação dos resultados por meio de escalogramas exige certa habilidade visual e familiaridade com o método apresentado. Para evitar erros óticos nesta pesquisa optou-se por trabalhar primeiramente com um grande número de dados colhidos em diferentes localizações e com séries históricas mais longas. Também se devem levar em consideração os efeitos de borda que acompanham os escalogramas (princípio da incerteza de Heisenberg). Assim, identificou-se um padrão de correlação acontecendo entre os anos de 2009 - 2010. As séries foram reduzidas e selecionaram-se os melhores resultados visuais. Demonstrou-se no artigo 11 escalogramas de coerência wavelet de regiões compreendidas entre 5 estados nordestinos: Maranhão, Piauí, Ceará, Rio Grande do Norte e Paraíba.

Todas as plataformas analisadas apresentam correlação. Embora esta correlação apresente variações nota-se que há um padrão de semelhança entre elas de acordo com localização. Uma analogia interessante é em relação à semelhança entre as correlações nas plataformas que estão localizadas no litoral e entre as plataformas que estão localizadas no interior do território pesquisado. Do que foi dito destaca-se a semelhança que há entre as plataformas localizadas nas cidades de João Pessoa, Natal e Fortaleza (figuras 2, 3 e 4, respectivamente). Elas apresentam correlações iguais no parâmetro escala em 310 - 850 e no parâmetro de tempo entre 400 - 1200. Todas elas estão localizadas em regiões litorâneas.

No interior, juntando em pares por requisito de semelhança e clareza, as correlações das plataformas em Cruzeta e Caracol (figuras 9 e 10, respectivamente) apresentam semelhança no tempo entre $400-$ 1500 e, embora na escala não fique tão exata, existe correlação na escala em 150 - 850, salvo os efeitos de borda.

Analogamente ao que foi dito pode-se obsevar as semelhanças entre as plataformas de Floriano e sobral (figuras 7 e 8); Quixeramobim e Seridó Caicó (figuras 5 e 6); Picos e Barra do Corda (figuras 11 e 12).

\section{Agradecimentos}

Ao $\mathrm{CNPq}$ pelo incentivo à pesquisa através da bolsa de iniciação científica PIBIC. Ao Instituto Na- 
cional de Meteorologia (INMET) pela disponibilização de dados meteorológicos e atendimento online. Aos professores orientadores pela troca de experiência e introdução da técnica.

\section{Referências Bibliográficas}

[1] E. A. LEITE. Análise Estatística de Padrões Sísmicos: Decomposição em Multiescala, Dissertação de Doutorado, Centro de Ciências Exatas e da Terra, Universidade Federal do Rio Grande do Norte, 2007.

[2] M. MISITI; Y. Misiti; G. Oppenheiim; J. M. Poggi. Wavelet Toolbox User's Guide: For Use with Matlab. The MathWorks; version 3. http://www.mathworks.com/access/helpdesk/help /pdf_doc/wavelet/wavelet_ug.pdf

[3] P. A. Morettin. Ondas e Ondaletas: Da Análise de Fourier à Análise de Ondaletas. São Paulo: Editora da Universidade de São Paulo, 1999.

[4] J. M. B. PROTÁZIO. Análise Wavelet Aplicada a Sinais Geofísicos, Dissertação de Mestrado, Faculdade de Engenharia Mecânica, Universidade Estadual de Campinas, 2002.

[5] H. B. SOARES. Análise e Classificação de Imagens de Lesões da Pele por Atributos de Cor, Forma e Textura Utilizando Máquina De Vetor De Suporte, Dissertação de Doutorado, Laboratório de Engenharia de Computação e Automação, Universidade Federal do Rio Grande do Norte, 2008.

[6] C. Torrence; G. P. Compo (1998). A Practical Guide to Wavelet Analysis. Program in Atmospheric and Oceanic Sciences. Vol. 79, No. 1. PP. 61-78.

[7] M. V. M. Varanis; R. Pederiva. Detecção de Correlação entre Séries Temporais Utilizando Espectro Cruzado Wavelet e Coerência Wavelet, $10^{\circ}$ Conferência Brasileira de Dinâmica, Controle e Aplicações - DINCON, Universidade Federal de Campinas, 2011. 